\title{
A Novel Approach to Reduce Traffic Chaos in Emergency and Evacuation Scenarios
}

\author{
Syed R. Rizvi, Stephan Olariu, Michele C. Weigle \\ Department of Computer Science \\ Old Dominion University \\ Norfolk, VA 23529 USA \\ \{srizvi, olariu, mweigle\}@cs.odu.edu
}

\author{
Mona E. Rizvi \\ Department of Computer Science \\ Old Dominion University \\ Norfolk, VA 23504 USA \\ mrizvi@nsu.edu
}

\begin{abstract}
This paper proposes a novel chaos reducing information dissemination approach for spatio-temporal traffic information related to first responders and evacuation scenarios using Vehicular Ad Hoc Networks (VANETs). In our approach, we provide an emergency vehicle path clearing technique. Therefore, traffic confusion and chaos is lowered on evacuation and emergency vehicle routes. Simulation results show that our approach works efficiently without fully relying on any message relaying infrastructure.
\end{abstract}

Keywords-VANET; Vehicular Ad Hoc Networks; Evacuation; Contraflow; Emergency Service Vehicles

\section{INTRODUCTION}

One of the major causes of traffic chaos is the confusion and failure to detect Emergency Service Vehicles (ESVs) (e.g. ambulances, police cars and fire trucks, etc.), which can lead to slow progress and even accidents involving the ESVs traveling towards their destinations. In addition to ESV scenarios, during an evacuation, there exists a tremendously challenging task for supply trucks to reach their destinations as quickly as possible and with minimal interruptions (see Fig. 1).

Vehicular Ad Hoc Networks (VANETs) have recently been proposed as a technology that can provide both drivers and passengers with a safe and enjoyable driving experience. Previous and current research and development [2, 4, 5, 6, 9] show that VANETs have recently gained much attention. Vehicles on the road, equipped with short and medium range wireless communication technology [14], form VANETs, whose main purpose is to exchange traffic safety and other security-related messages. VANETs can be used for many applications with vehicle-to-vehicle (V2V) and vehicle-toinfrastructure (V2I) communication. Some of the significant applications of VANETs are road safety applications including collision and other safety warning systems $[3,15,10]$, driver convenience and information systems and, in the future, intelligent traffic management systems.

The idea presented in this paper is that if all motorists are able to receive information through a broadcast from moving ESV(s), the algorithm can detect if, when, and where the ESV and the motorist will meet and thus provide advanced warning to the driver of the approaching ESV. This approach can also be employed in an evacuation where ESVs will be the supply trucks that are required to reach various locations along the evacuation path. Consequently, our approach enhances the effectiveness of existing evacuation plans.

\section{RELATED WORK}

The sudden appearance of an ESV en route to an emergency can be critically disruptive to nearby vehicles as individual drivers maneuver to get out of the way. ESVs operating in higher congestion levels are at higher risk for involvement in accidents and are subject to unpredictable delays in reaching the scene of a fire or crash. EMS personnel in the United States have an estimated fatality rate of 12.7 per 100,000 workers, more than twice the national average [8]. Our VANET-based approach primarily assists emergency response units in safely reaching the incident location as fast as possible.

In the U.S., the FCC has already approved $75 \mathrm{MHz}$ of spectrum for vehicles to communicate among themselves (V2V communications) and with road-side infrastructure (V2I communication), known as Dedicated Short Range Communication (DSRC). This DSRC system is expected to be the first wide-scale VANET in North America. In Japan, two DSRC standards have been adopted, and the Japanese automobile manufacturers are coordinating with Japanese governments on a large-scale Advanced Safety Vehicle Project [6]. In Europe, several projects, such as the Fleetnet project in Germany [4], have been conducted (see Table 1). Although research has been done on evacuation using infrastructurebased Intelligent Transportation Systems (ITS) [12], less attention has been given towards proposing a solution with a combination of VANET and evacuation rescue efforts.

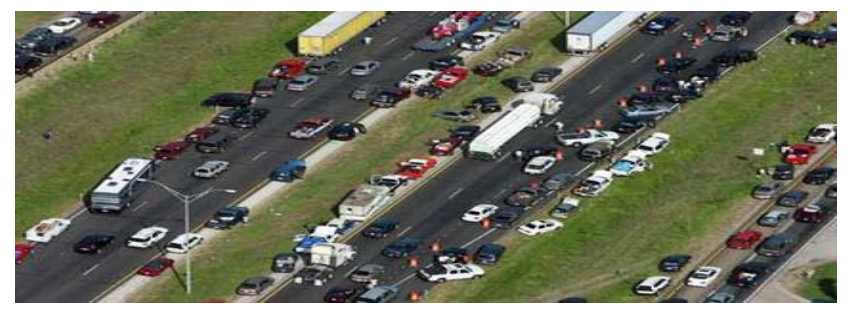

Figure 1. Cars sit broken down or without fuel by the side of the road in Huntsville, Texas. Trucks filled with life-saving relief supplies are unable to reach their destinations [7]. 
TABLE I. SOME TRAFFIC MONITORING MANAGEMENTS SYSTEMS

\begin{tabular}{|l|l|}
\hline \multicolumn{1}{|c|}{ Projects } & \multicolumn{1}{c|}{ Highlights } \\
\hline $\begin{array}{l}\text { Pilot project in } \\
\text { Atlanta [5] }\end{array}$ & $\begin{array}{l}\text { Uses a fleet of 500 "traffic spies" equipped with } \\
\text { onboard sensors, computer and GPS unit. }\end{array}$ \\
\hline $\begin{array}{l}\text { Advanced Cruise- } \\
\text { Assistant Highway } \\
\text { Systems (AHS) [1] }\end{array}$ & $\begin{array}{l}\text { Assist at reducing traffic accidents, enhancing } \\
\text { safety, improving transportation efficiency, and } \\
\text { reducing the operational involvement of drivers. }\end{array}$ \\
\hline $\begin{array}{l}\text { Vehicle Information } \\
\text { and Communication }\end{array}$ & $\begin{array}{l}\text { Allow drivers to obtain road and traffic } \\
\text { information in real-time. }\end{array}$ \\
Systems (VICS) [6] & \\
\hline FleetNet [4], & $\begin{array}{l}\text { FleetNet attempts to develop wireless multi-hop } \\
\text { ad hoc networks for inter-vehicle }\end{array}$ \\
AutoNet [2], and & communication to improve safety, and provide \\
Path [9] & drivers with up-to-date information on traffic, \\
& weather, etc. \\
\hline
\end{tabular}

One means to offset the effects of congestion is the installation of Emergency Vehicle Preemption (EVP) equipment at signalized intersections [16]. Using ITS to provide ESVs a green light at intersections can reduce driver confusion, reduce conflicts, and improve emergency response times. However, EVP does not work in evacuation scenarios where there are no traffic signals, such as on highways or in rural areas.

In this paper, we propose a VANET-based approach that reduces the chaos and confusion among evacuees and first responders. The key ingredient of our approach is the prediction based on the spatio-temporal character of ESVs, which broadens the ability of ESVs to be used to their maximum effectiveness.

\section{EVACUATION ENVIRONMENT}

\section{A. Evacuation Issues}

Information about traffic flow rates, about the location of incidents that block lanes or that slow traffic, and about weather conditions and flood levels is critical. ITS technologies can be used to gather this information; however, the concentration of ITS deployment is in urban areas, and evacuation travel mostly occurs in rural areas [12]. In our approach, since we do not fully rely on any message relaying infrastructure, our solution is well suited for rural areas.

\section{B. Suggested Solutions}

Preliminary studies have shown that contraflow strategies can increase the outbound volume by about 70 percent [11]. Recent evacuations have highlighted a number of difficulties with contraflow. Many motorists were not allowed to exit the highways despite a variety of needs. Additionally, fuel and other urgent supplies need to be positioned prior to employing contraflow, or efforts to supply aid to the stricken or threatened areas would slow evacuation efforts.

\section{VANET System Model}

In our approach, all vehicles act as information servers relaying information for the VANET. We do not make use of any access points on the road side or depend upon a collection of location servers.

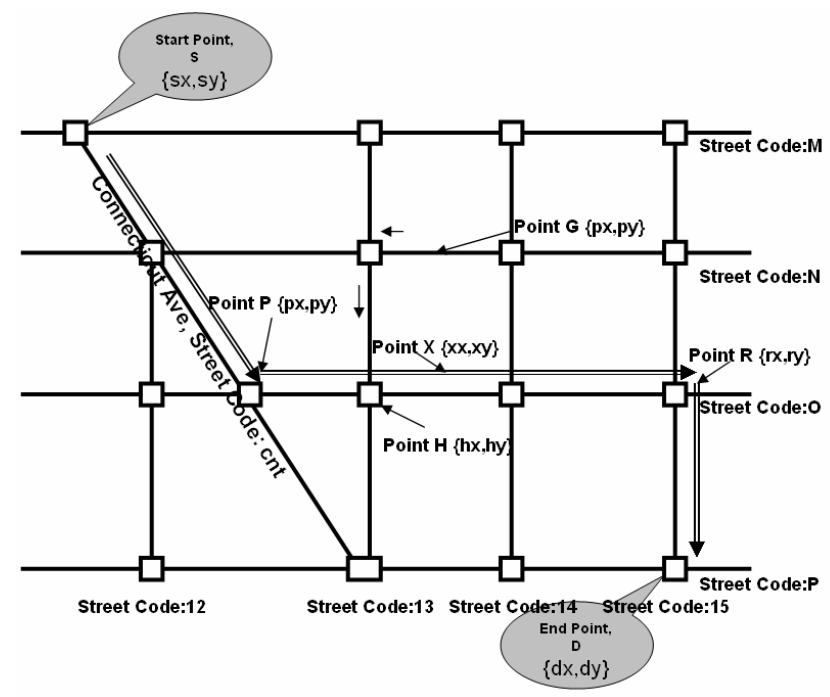

Figure 2. Illustration of the elements of an ESV route and the computation of time $t_{x}$.

We assume that every vehicle has a digital map and knows its geographical position and heading through a Global Positioning System (GPS) receiver. The building blocks of our approach are:

- Resources: ESV etc;

- Reports: Information sent by resources. V2V communication is used to relay reports;

- Broadcasting: We exploit broadcasting techniques for information propagation and take the spatio-temporal character of reports into account;

- Selection strategies based on relevance: The spatial distribution of information is controlled through a selection strategy.

\section{TRafFic ChaOs Reduction Approach}

\section{A. ESV Route Traffic Chaos Reduction Approach}

When an emergency service is called, one or more ESVs can be dispatched to a destination. Since the destination is known at the time that the emergency service personnel are dispatched, the route from a starting point (e.g. hospital) to a destination point (e.g. accident location) can be traced on a digital map in an ESV equipped with a GPS unit. We call this route the ESV Route. The ESV periodically (with a pre-defined broadcast interval) broadcasts a report containing the following information:

- Unique ID of ESV (denoted by esv_id),

- Type of ESV (e.g. ambulance, police car or fire truck) (denoted by $\theta$ ),

- Start and end points (denoted by $\{s x, s y\}$ and $\{d x, d y\}$, respectively),

- $\quad$ Route Code (denoted $\Omega$ ), 
- Tentative average speed of ESV on the ESV Route (denoted by $v_{e}$ ),

- Current ESV location coordinates and current time (denoted by $\{c x, c y\}$, and $t_{c}$ respectively),

- Timestamp denoting the time the report was sent by the ESV (denoted by 6 ).

Each ESV is assigned a unique ID, esv id, to distinguish reports sent by different ESVs within an area. The type of ESV, $\theta$, could help other motorists prepare for the kind of ESV for which they will have to clear the way. Start and end point coordinates along with the route information are vital in our approach. Each street is assigned a unique street ID. Vehicles are preloaded with digital maps that contain the street IDs for an entire city, or one or more states. The Route Code for an ESV Route is defined as a sequence of street codes with initial coordinates of each street. The street codes are ordered from the stating point to the end point. For example, in Fig. 2, a solid line represents a street, and a small square at a crossing point of streets represents an intersection. S and D are the start and end points respectively for an ESV Route. The example route shown in Fig. 2 can be traced in the following order:

(1) S to P (i.e. Connecticut Ave., Street Code: cnt),

(2) P to R (i.e. Street Code: O), and

(3) R to D (i.e. Street Code: 15).

The starting point of each street on this ESV route can also be easily obtained:

(1) Street Code: cnt - Point S $\{\mathrm{sx}, \mathrm{sy}\}$,

(2) Street Code: $\mathrm{O}-$ Point $\mathrm{P}\{\mathrm{px}, \mathrm{py}\}$, and

(3) Street Code: 15 - Point R \{rx,ry\}.

Therefore, the Route Code $\Omega=\{(s x, s y) / c n t,(p x, p y) / O$, $(r x, r y) / 15\}$. The direction of the ESV route, is determined by the order of streets.

Any vehicle that receives $\{s x, s y\},\{d x, d y\}$, and $\Omega$ from an ESV can reconstruct the actual ESV Route on its digital map by matching the street codes to $\Omega$. The tentative average speed of an ESV on a route $\Omega$ is a predicted average speed for the ESV. The current location coordinates and current time, $\{c x, c y\}$ and $t_{c}$, provide information about when and where the ESV was located when the report was sent. The timestamp along with the current location helps with sorting the reports for their relevance, as will be explained later. Each vehicle stores its current average speed, $v_{v}$, calculated during a past short period of time. The value of $v_{v}$ is periodically updated. Assume that at time $t_{g}$, a vehicle is located at point G on the street $\mathrm{N}$ (see Fig. 2 ). We assume that at time $t_{x}$, the ESV and the vehicle will meet at point X on the ESV Route. With the ESV Route known to the vehicle, let $\mathrm{H}$ be the point where the vehicle will meet the ESV Route. Let $d_{v}$ be the distance on the vehicle's route between $\mathrm{G}$ and $\mathrm{H}$. Let $d_{e}$ be the distance on the ESV Route between $\{c x, c y\}$ and $H$. In Fig. 2, point $X$ will be on the left side of $\mathrm{H}$ if the vehicle is moving in the opposite direction of the ESV Route. We call this Opposite Direction Movement (ODM). Similarly, point $\mathrm{X}$ will be on the right side of $\mathrm{H}$ if the vehicle is moving in the same direction as the ESV Route. We call this Same Direction Movement (SDM). Computation performed using the average ESV speed $\left(v_{e}\right)$, ESV location coordinates $(\{c x, c y\})$, and current time $\left(t_{c}\right)$ obtained from the report sent by an ESV yields:

- $t_{x}=\left(\left(d_{e}-d_{v}\right)+\left(v_{e} t_{c}-v_{v} * t_{g}\right)\right) /\left(v_{e}-v_{v}\right)$ for SDM

- $t_{x}=\left(\left(d_{e}+d_{v}\right)+\left(v_{e}{ }^{*} t_{c}+v_{v}{ }^{*} t_{g}\right)\right) /\left(v_{e}+v_{v}\right)$ for ODM

With this computed $t_{x}$, a short period of clearing path time, $t_{c l r}$ (30 sec or $1 \mathrm{~min}$ before the ESV meets the other vehicle), which is the time range during which the motorist should clear the way for the ESV, can be provided to the motorist. Note that the value of $t_{x}$ is dynamic since $v_{e}$ and $v_{v}$ can both change.

\section{B. Selection Strategy based on Relevance}

Relevance describes the degree of applicability of a received report. The relevance of a resource report $R$ is calculated through a relevance function $r(R)=-\sigma-d_{e} / v_{e}[13]$. During a vehicle's trip, it receives resource reports from resources or other vehicles. Periodically, reports in a vehicle's database are sorted according to their relevance. The most relevant report is used for computing $t_{x}$. For a vehicle, if a point $\mathrm{H}$ (see Fig. 2) is forecast, i.e. the vehicle will travel on an ESV Route in the future, then $t_{x}$ is computed, otherwise the most relevant report is rebroadcast for other vehicles.

\section{Evacuation Contraflow Traffic Chaos Reduction}

During an evacuation, the path of supply trucks traveling in the opposite direction of the evacuating contraflow traffic is severely impeded. We can employ our ESV Route Traffic Chaos Reduction Approach (described in Section V, A) during an evacuation to clear the way for these needed supply trucks.

\section{Simulation RESUlts}

We synthetically generated and moved vehicles within a terrain size of $4000 \mathrm{~m} \times 4000 \mathrm{~m}$ (see to Fig. 2) with one source (S) and one destination (D). Nodes move in accordance to a realistic mobility and traffic model following city street structures. The model is realistic because we take into account different traffic densities. One ESV is considered to travel from $\mathrm{S}$ to $\mathrm{D}$ in each scenario. Each vehicle needs to decide turning directions at the intersection with a smooth deceleration and acceleration pattern. Nodes move between $5-10 \mathrm{~m} / \mathrm{s}$ and the ESV moves between $10-15 \mathrm{~m} / \mathrm{s}$. The broadcast interval is 5 seconds. Transmission range of vehicles is $50-300 \mathrm{~m}$. Transmission range of the ESV is always $300 \mathrm{~m}$. All the key parameters of our simulation are summarized in the Table 2. We show the mean data success ratio over the various traffic densities and transmission range in Fig. 3 and Fig. 4, respectively. The data success rate is defined as the ratio of the number of vehicles on the ESV route that have received the message within a certain clearing path time to the total number of vehicles on the ESV route. Fig. 3-4 shows our results. Note that the ideal case of a high density of 75 vehicles per square $\mathrm{km}$ or a high transmission range of $300 \mathrm{~m}$ gives a data success rate value of 1 . We found that by increasing the values of the resource report size, no performance improvement is obtained. 
TABLE II. SIMULATION SETUP

\begin{tabular}{|l|l|}
\hline \multicolumn{1}{|c|}{ Parameters } & \multicolumn{1}{c|}{ Settings } \\
\hline Resource report size & 64 bytes/ Variable \\
\hline Baseline bandwidth & $5 \mathrm{Mbps}$ \\
\hline Packet sending rate & 5 seconds \\
\hline Number of vehicles & variable \\
\hline Transmission range & $50 \mathrm{~m}-300 \mathrm{~m}$ \\
\hline Terrain size & $4000 \mathrm{~m} \mathrm{X} \mathrm{4000} \mathrm{m}$ \\
\hline Additional map rim & $500 \mathrm{~m}$ \\
\hline Average vehicle speed & $5-10 \mathrm{~m} / \mathrm{s}$ \\
\hline Average ESV speed & $10-15 \mathrm{~m} / \mathrm{s}$ \\
\hline MAC protocol & IEEE $802.11 \mathrm{DCF}$ \\
\hline Simulation time & $500 \mathrm{~s}$ \\
\hline
\end{tabular}

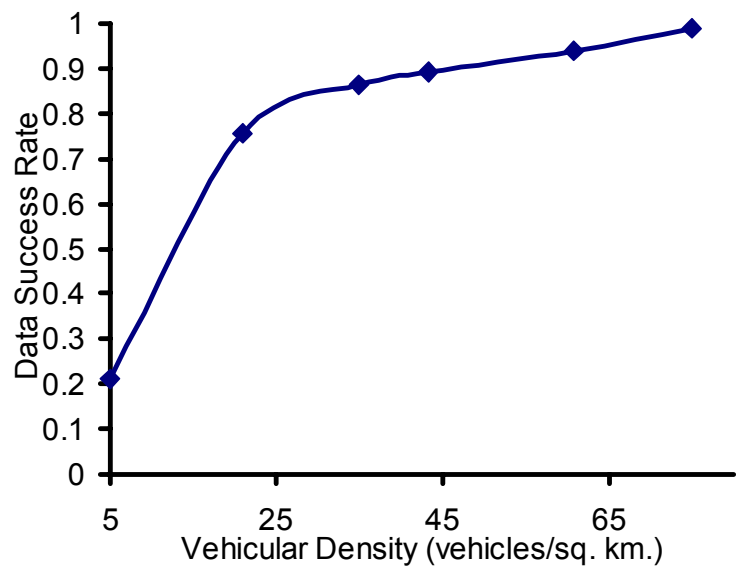

Figure 3. Data success rate as a function of the number of nodes. Resource report size $=64$ bytes; transmission range $=150 \mathrm{~m}$.

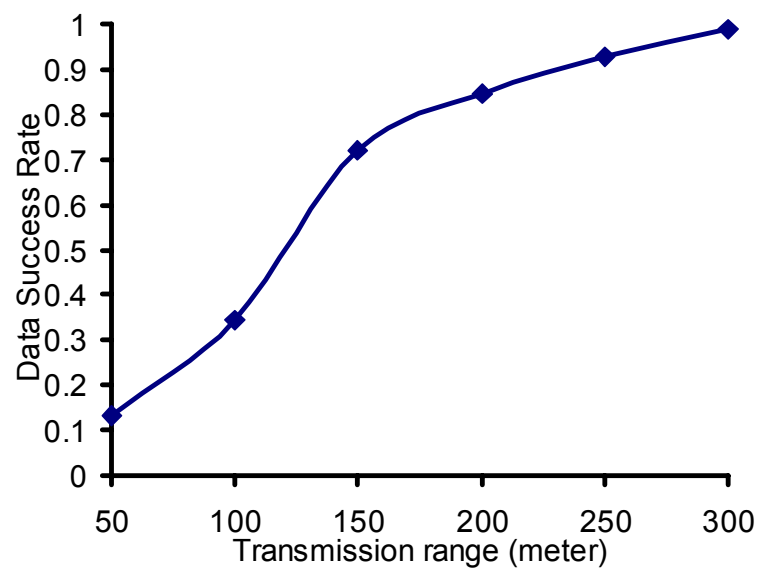

Figure 4. Data success rate as a function of the transmission range. Resource report size $=64$ bytes; vehicular density $=40$ vehicles $/$ sq.. $\mathrm{km}$.
In Fig. 3, the data success rate of our approach is shown as a function of the number of nodes (vehicular density in vehicles per square $\mathrm{km}$ ) in the network. As is expected, increasing the density will improve the success rate. For our VANET-based infrastructureless system, this density dependency helps in the emergency and evacuation scenarios for the ESVs operating in higher congestion levels, which are otherwise at higher risk for involvement in accidents and unpredictable delays. In Fig. 4, increasing the transmission range of each vehicle increases the success rate. Thus, as the transmission range and the vehicular density increase, the ESV route information is propagated more rapidly and reaches the concerned vehicles sooner, giving the motorists a higher probability of getting a clear picture of the situation.

\section{CONCLUSIONS}

Emergency Service Vehicles operating in highly congested areas, during evacuation as well as non-evacuation conditions, are at higher risk for involvement in accidents and are subject to unpredictable delays in reaching the stricken or threatened area. Although the transportation infrastructure cannot accommodate evacuation-level traffic demand, efficiency can increase with our approach, and strategic planning and management will improve. Our VANET-based approach can be coupled with EVP in order to feed appropriate ESV Route information to the EVP infrastructure from the report broadcasts relayed through the vehicles. Based on our introduced approach and the obtained results from our realistic mobility and traffic model based simulation study, we conclude that applications like the Traffic Chaos Reduction for emergency scenarios are potentially attractive rollout applications for VANETs.

\section{FUTURE WORK}

Although a state's network of public radio stations can broadcast traffic and shelter information during evacuations, one of the main problems for motorists is filtering the information. An evacuee would only like to know what shelters are currently available near his or her current location and would likely not be interested in knowing the status of all shelters in a city. Real-time filtered information about the availability and location of shelters, gas stations, etc. can be utilized by the evacuating motorists. This would avoid the wastage of effort, time and fuel in searching for useful resources.

Our approach can be extended if parking places, gas stations, hospitals, schools, and shelters periodically broadcast reports that include the following information:

- type of the resource,

- availability of the resource,

- location coordinates of the resource, and

- timestamp denoting the time the report was sent.

The current location along with the timestamp would help with sorting the reports. Note that $v_{e}$ will be replaced by $v_{v}$ for stationary resources. These reports will be updated as resource availability changes. The information can be easily filtered 
with respect to a vehicle's position. Optimization of bandwidth consumption is also left for future work.

\section{REFERENCES}

[1] AHS: Advanced Cruise-Assist Highway System Research Association, www.ahsra.or.jp.

[2] AutoNet: Ad hoc Peer to Peer Information Technology for Traffic Networks, www.its.uci.edu/monally/mgmautonet.htm.

[3] I. Chisaliat and N. Shahmehri, "A Peer-to-Peer Approach to Vehicular Communication for the Support of Traffic Safety Applications", in ITSC IEEE, 2002.

[4] Fleetnet, www.et2.tu-harburg.du/fleetnet/index.html.

[5] E. Guizzo, "Network of Traffic Spies Built into Cars in Atlanta", IEEE Spectrum online, 2004.

[6] Japanese Ministry of Land, Infrastructure and Transport, Road Bureau ITS, www.its.go.jp/ITS/index.html.

[7] K. Li, "Evacuation Chaos, Death as Hurricane Surges On", The Age, Sept 25, 2005.

[8] B. Maguire, K. Hunting, G. Smith, N. Levick, "Occupational Fatalities in Emergency Medical Services: A Hidden Crisis", Ann Emerg Med 2002; 40:625-32.

[9] Partners for Advanced Transit and Highway (PATH), www.path.eecs.berkeley.edu.

[10] Chr. Passmann, Ch. Benzel, and R. Meschenmoser, "Wireless Vehicle To Vehicle Warning Systems", SAE 2000 World Congress, Detroit, Michigan, March 2000.

[11] Southeast United States Hurricane Evacuation Traffic Study: Exec Summary.FEMA, Washington, D.C., 2000.

[12] B. Wolshon and B. H. Meehan, "Emergency Evacuation - Ensuring Safe and Efficient Transportation out of Endangered Areas", TR News Transportation Research Board of the National Academies, 224:3-9, Jan 2003.

[13] O. Wolfson, B. Xu, and H. Yin, "Reducing Resource Discovery Time by Spatio-Temporal Information in Vehicular Ad-Hoc Networks", In Proceedings of the Second ACM International Workshop on VANET, pages 91-92, Cologne, Germany, 2005.

[14] U. Varsheney, "Vehicular Mobile Commerce", IEEE Computer Magazine Online, December 2004.

[15] J. Yin et al "Performance Evaluation of Safety Applications over DSRC Vehicular Ad Hoc Networks", Proceedings of the first ACM workshop on VANETs, Philadelphia, 2004.

[16] I. Yun, M. Best, and B. Park, "Evaluation of Emergency Vehicle Preemption Strategies on a Coordinated Actuated Signal System Using Hardware-in-the-Loop Simulation", in Transportation Research Board 86th Annual Meeting Paper \#07-2415, Washington DC, 2007. 